L. Aharouch, E. Azroul and M. Rhoudaf (Fès)

\title{
NONLINEAR UNILATERAL PROBLEMS IN ORLICZ SPACES
}

Abstract. We prove the existence of solutions of the unilateral problem for equations of the type $A u-\operatorname{div} \phi(u)=\mu$ in Orlicz spaces, where $A$ is a Leray-Lions operator defined on $\mathcal{D}(A) \subset W_{0}^{1} L_{M}(\Omega), \mu \in L^{1}(\Omega)+$ $W^{-1} E_{\bar{M}}(\Omega)$ and $\phi \in C^{0}\left(\mathbb{R}, \mathbb{R}^{N}\right)$.

1. Introduction. Let $\Omega$ be a bounded domain in $\mathbb{R}^{N}$ with the segment property. Consider the following nonlinear Dirichlet problem:

$$
A u-\operatorname{div} \phi(u)=\mu,
$$

where $A u=-\operatorname{div} a(x, u, \nabla u)$ is a Leray-Lions operators defined on its domain $\mathcal{D}(A)=\left\{u \in W_{0}^{1} L_{M}(\Omega): a(x, u, \nabla u) \in\left(L_{\bar{M}}(\Omega)\right)^{N}\right\}$ into $W^{-1} E_{\bar{M}}(\Omega)$, with $M$ an $N$-function and $\phi \in \mathcal{C}^{0}\left(\mathbb{R}, \mathbb{R}^{N}\right)$. The right-hand side $\mu$ is assumed to belong to $L^{1}(\Omega)+W^{-1} E_{\bar{M}}(\Omega)$.

In the variational case (i.e. where $\mu \in W^{-1} E_{\bar{M}}(\Omega)$ ), J.-P. Gossez and V. Mustonen [14] solved (1.1) in the case where $\phi=0$. The case where $\mu \in L^{1}(\Omega)$ is treated in $[5,6]$.

In [6], the authors deal with the case $\phi=0$. They prove the existence and uniqueness of solution for the associated unilateral problem but under some restriction on the $N$-function $M$ (the $\Delta_{2}$-condition), and in [5] they etablish the existence of an entropy solution of (1.1) without any restriction on $M$.

It is our purpose in this paper to prove the existence of solution for the unilateral problem associated to the equation (1.1) in the setting of Orlicz spaces for general $N$-functions $M$.

Let us also mention the works of Elmahi and Meskine [11, 12] who studied the existence of solutions for equations of the form $-\operatorname{div} a(x, u, \nabla u)+$

2000 Mathematics Subject Classification: 35J25, 35J60.

Key words and phrases: Orlicz-Sobolev spaces, boundary value problems, truncations, unilateral problems. 
$g(x, u, \nabla u)=f$ where $g$ is a nonlinearity having natural growth and satisfying the sign condition, and the term $f$ belongs either to $W^{-1} E_{\bar{M}}(\Omega)$ or to $L^{1}(\Omega)$. These results are generalized in $[2,3]$.

Let us briefly summarize the contents of the paper. After a section devoted to developing the necessary preliminaries, we introduce some technical lemmas (Section 2). In Section 3, we give our main result and we prove it in Section 4.

2. Preliminaries and some technical lemmas. In this section we list briefly some definitions and well known facts about $N$-functions and Orlicz-Sobolev spaces. Standard references are [1, 7, 15].

2.1. Let $M: \mathbb{R}^{+} \rightarrow \mathbb{R}^{+}$be an $N$-function, i.e. $M$ is continuous, convex, with $M(t)>0$ for $t>0, M(t) / t \rightarrow 0$ as $t \rightarrow 0$ and $M(t) / t \rightarrow \infty$ as $t \rightarrow \infty$.

Equivalently, $M$ admits a representation $M(t)=\int_{0}^{t} a(s) d s$ where $a$ : $\mathbb{R}^{+} \rightarrow \mathbb{R}^{+}$is a nondecreasing, right continuous function, with $a(0)=0$, $a(t)>0$ for $t>0$ and $a(t) \rightarrow \infty$ as $t \rightarrow \infty$.

The $N$-function $\bar{M}$ conjugate to $M$ is defined by $\bar{M}(t)=\int_{0}^{t} \bar{a}(s) d s$, where $\bar{a}: \mathbb{R}^{+} \rightarrow \mathbb{R}^{+}$is given by $\bar{a}(t)=\sup \{s: a(s) \leq t\}$.

The $N$-function $M$ is said to satisfy the $\Delta_{2}$-condition if, for some $k$,

$$
M(2 t) \leq k M(t) \quad \forall t \geq 0 .
$$

It is readily seen that this is the case if and only if for every $r>0$ there exists a positive constant $k=k(r)$ such that for all $t>0$,

$$
M(r t) \leq k M(t) \quad \forall t \geq 0 .
$$

If (2.1) and (2.2) hold only for $t \geq t_{0}$ for some $t_{0}>0$ then $M$ is said to satisfy the $\Delta_{2}$-condition near infinity.

We extend $N$-functions to even functions on all of $\mathbb{R}$.

Moreover, we have the following Young inequality:

$$
\forall s, t \geq 0, \quad s t \leq M(t)+\bar{M}(s) .
$$

Let $P$ and $Q$ be two $N$-functions. We say that $P$ grows essentially less rapidly than $Q$ near infinity, written $P \ll Q$, if for every $\varepsilon>0, P(t) / Q(\varepsilon t) \rightarrow 0$ as $t \rightarrow \infty$. This is the case if and only if $\lim _{t \rightarrow \infty} Q^{-1}(t) / P^{-1}(t)=0$.

2.2. Let $M$ be an $N$-function and $\Omega \subset \mathbb{R}^{N}$ be an open and bounded set. The Orlicz class $\mathcal{K}_{M}(\Omega)$ (resp. the Orlicz space $L_{M}(\Omega)$ ) is defined as the set of (equivalence classes of) real-valued measurable functions $u$ on $\Omega$ such that

$$
\int_{\Omega} M(u(x)) d x<\infty \quad\left(\text { resp. } \int_{\Omega} M(u(x) / \lambda) d x<\infty \text { for some } \lambda>0\right) .
$$


$L_{M}(\Omega)$ is a Banach space under the norm

$$
\|u\|_{M, \Omega}=\inf \left\{\lambda>0: \int_{\Omega} M(u(x) / \lambda) d x \leq 1\right\}
$$

and $\mathcal{K}_{M}(\Omega)$ is a convex subset of $L_{M}(\Omega)$ but not necessarily a linear space.

The closure in $L_{M}(\Omega)$ of the set of bounded measurable functions with compact support in $\bar{\Omega}$ is denoted by $E_{M}(\Omega)$.

The dual space of $E_{M}(\Omega)$ can be identified with $L_{\bar{M}}(\Omega)$ by means of the pairing $\int_{\Omega} u v d x$, and the dual norm of $L_{\bar{M}}(\Omega)$ is equivalent to $\|\cdot\|_{\bar{M}, \Omega}$.

Let $X$ and $Y$ be arbitrary Banach spaces with a bilinear bicontinuous pairing $\langle,\rangle_{X, Y}$. We say that a sequence $\left\{u_{n}\right\} \subset X$ converges to $u \in X$ with respect to the topology $\sigma(X, Y)$, written $u_{n} \rightarrow u(\sigma(X, Y))$ in $X$, if $\left\langle u_{n}, v\right\rangle \rightarrow\langle u, v\rangle$ for all $v \in Y$. For example, if $X=L_{M}(\Omega)$ and $Y=L_{\bar{M}}(\Omega)$, then the pairing is defined by $\langle u, v\rangle=\int_{\Omega} u(x) v(x) d x$ for all $u \in X, v \in Y$.

2.3. We now turn to the Orlicz-Sobolev space $W^{1} L_{M}(\Omega)$ [resp. $\left.W^{1} E_{M}(\Omega)\right]$, which is the space of all functions $u$ such that $u$ and its distributional derivatives of order 1 lie in $L_{M}(\Omega)$ [resp. $\left.E_{M}(\Omega)\right]$. It is a Banach space under the norm

$$
\|u\|_{1, M}=\sum_{|\alpha| \leq 1}\left\|D^{\alpha} u\right\|_{M}
$$

Thus, $W^{1} L_{M}(\Omega)$ and $W^{1} E_{M}(\Omega)$ can be identified with subspaces of the product of $N+1$ copies of $L_{M}(\Omega)$. Denoting this product by $\prod L_{M}$, we will use the weak topologies $\sigma\left(\prod L_{M}, \prod E_{\bar{M}}\right)$ and $\sigma\left(\prod L_{M}, \prod L_{\bar{M}}\right)$.

The space $W_{0}^{1} E_{M}(\Omega)$ is defined as the (norm) closure of the Schwartz space $\mathcal{D}(\Omega)$ in $W^{1} E_{M}(\Omega)$, and $W_{0}^{1} L_{M}(\Omega)$ as the $\sigma\left(\prod L_{M}, \prod E_{\bar{M}}\right)$ closure of $\mathcal{D}(\Omega)$ in $W^{1} L_{M}(\Omega)$.

We say that a sequence $\left\{u_{n}\right\} \subset L_{M}(\Omega)$ converges to $u \in L_{M}(\Omega)$ in the modular sense, and write $u_{n} \rightarrow u(\bmod )$ in $L_{M}(\Omega)$, if for some $\lambda>0$,

$$
\int_{\Omega} M\left(\left|u_{n}(x)-u(x)\right| / \lambda\right) d x \rightarrow 0 \quad \text { as } n \rightarrow \infty .
$$

If $M$ satisfies the $\Delta_{2}$-condition (near infinity only when $\Omega$ has finite measure), then modular convergence coincides with norm convergence (see [15]).

We say that a sequence $\left\{u_{n}\right\} \subset W^{1} L_{M}(\Omega)$ converges to $u \in W^{1} L_{M}(\Omega)$ in the modular sense, and write $u_{n} \rightarrow u(\bmod )$ in $W^{1} L_{M}(\Omega)$, if there exists $\lambda>0$ such that

$$
\int_{\Omega} M\left(\left|D^{\alpha} u_{n}(x)-D^{\alpha} u(x)\right| / \lambda\right) d x \rightarrow 0 \quad \text { for all }|\alpha| \leq 1 \text { as } n \rightarrow \infty .
$$

2.4. Let $W^{-1} L_{\bar{M}}(\Omega)$ [resp. $W^{-1} E_{\bar{M}}(\Omega)$ ] denote the space of distributions on $\Omega$ which can be written as sums of derivatives of order $\leq 1$ of functions in $L_{\bar{M}}(\Omega)$ [resp. $\left.E_{\bar{M}}(\Omega)\right]$. It is a Banach space under the usual quotient norm. 
We recall some lemmas introduced in [7] which will be used later.

Lemma 2.1. Let $F: \mathbb{R} \rightarrow \mathbb{R}$ be uniformly Lipschitzian with $F(0)=0$. Let $M$ be an $N$-function and let $u \in W^{1} L_{M}(\Omega)$ (resp. $u \in W^{1} E_{M}(\Omega)$ ). Then $F(u) \in W^{1} L_{M}(\Omega)$ (resp. $F(u) \in W^{1} E_{M}(\Omega)$ ). Moreover, if the set $D$ of discontinuity points of $F^{\prime}$ is finite, then

$$
\frac{\partial}{\partial x_{i}} F(u)= \begin{cases}F^{\prime}(u) \frac{\partial}{\partial x_{i}} u & \text { a.e. in }\{x \in \Omega: u(x) \notin D\}, \\ 0 & \text { a.e. in }\{x \in \Omega: u(x) \in D\} .\end{cases}
$$

Lemma 2.2. Let $F: \mathbb{R} \rightarrow \mathbb{R}$ be uniformly Lipschitzian with $F(0)=0$. Suppose that the set of discontinuity points of $F^{\prime}$ is finite. Let $M$ be an $N$-function. Then the mapping $T_{F}: W^{1} L_{M}(\Omega) \rightarrow W^{1} L_{M}(\Omega)$ defined by $T_{F}(u)=F(u)$ is sequentially continuous with respect to the weak ${ }^{*}$ topology $\sigma\left(\prod L_{M}, \prod E_{\bar{M}}\right)$.

We now give the following lemma which concerns operators of the Nemytskiı̌ type in Orlicz spaces (see [7]).

LEMMA 2.3. Let $\Omega$ be an open subset of $\mathbb{R}^{N}$ with finite measure. Let $M, P$ and $Q$ be $N$-functions such that $Q \ll P$, and let $f: \Omega \times \mathbb{R} \rightarrow \mathbb{R}$ be a Carathéodory function such that, for a.e. $x \in \Omega$ and all $s \in \mathbb{R}$,

$$
|f(x, s)| \leq c(x)+k_{1} P^{-1} M\left(k_{2}|s|\right),
$$

where $k_{1}, k_{2}$ are real constants and $c \in E_{Q}(\Omega)$. Then the Nemytskiu operator $N_{f}$ defined by $N_{f}(u)(x)=f(x, u(x))$ is strongly continuous from $\mathcal{P}\left(E_{M}(\Omega), 1 / k_{2}\right)=\left\{u \in L_{M}(\Omega): d\left(u, E_{M}(\Omega)\right)<1 / k_{2}\right\}$ into $E_{Q}(\Omega)$.

We introduce the function spaces we will need later.

For an $N$-function $M, \mathcal{T}_{0}^{1, M}(\Omega)$ is defined as the set of measurable functions $u: \Omega \rightarrow \mathbb{R}$ such that for all $k>0$ the truncated functions $T_{k}(u)$ are in $W_{0}^{1} L_{M}(\Omega)$, where $T_{k}(s)=\max (-k, \min (k, s))$.

We give the following lemma which is a generalization of [4, Lemma 2.1] to Orlicz spaces and whose proof is a slight modification of the one in the $L^{p}$ case.

LEMma 2.4. For every $u \in \mathcal{T}_{0}^{1, M}(\Omega)$, there exists a unique measurable function $v: \Omega \rightarrow \mathbb{R}^{N}$ such that

$\nabla T_{k}(u)=v \chi_{\{|u|<k\}}, \quad$ almost everywhere in $\Omega$, for every $k>0$.

We will call $v$ the gradient of $u$, and write $v=\nabla u$.

LEMmA 2.5. Let $\lambda \in \mathbb{R}$ and let $u, v \in \mathcal{T}_{0}^{1, M}(\Omega)$ be finite almost everywhere. Then

$$
\nabla(u+\lambda v)=\nabla u+\lambda \nabla v \quad \text { a.e. in } \Omega,
$$

where $\nabla$ is the gradient introduced in Lemma 2.4. 
The proof of this lemma is similar to the proof of [10, Lemma 2.12] in the $L^{p}$ case.

Below, we will use the following technical lemmas.

Lemma $2.6([7])$. Let $f_{n}, f, \gamma \in L^{1}(\Omega)$ be such that

(i) $f_{n} \geq \gamma$ a.e. in $\Omega$,

(ii) $f_{n} \rightarrow f$ a.e. in $\Omega$,

(iii) $\int_{\Omega} f_{n}(x) d x \rightarrow \int_{\Omega} f(x) d x$.

Then $f_{n} \rightarrow f$ strongly in $L^{1}(\Omega)$.

Lemma 2.7 ([5]). Let $\Omega$ be an open bounded subset of $\mathbb{R}^{N}$ with the segment property. If $u \in W_{0}^{1} L_{M}(\Omega)$, then

$$
\int_{\Omega} \operatorname{div} u d x=0 .
$$

\section{Statement of main results}

3.1. Basic assumptions. Let $\Omega$ be an open bounded subset of $\mathbb{R}^{N}$, $N \geq 2$, with the segment property and $M$ be an $N$-function.

Given a measurable obstacle function $\psi: \Omega \rightarrow \overline{\mathbb{R}}$, we consider the set

$$
K_{\psi}=\left\{u \in W_{0}^{1} L_{M}(\Omega): u \geq \psi \text { a.e. in } \Omega\right\} .
$$

This convex set is sequentially $\sigma\left(\prod L_{M}, \prod E_{\bar{M}}\right)$ closed in $W_{0}^{1} L_{M}(\Omega)$ (see [14]). We now state our hypotheses on the differential operator $A$ defined by

$$
A u=-\operatorname{div}(a(x, u, \nabla u)) .
$$

$\left(A_{1}\right) \quad a(x, s, \xi): \Omega \times \mathbb{R} \times \mathbb{R}^{N} \rightarrow \mathbb{R}^{N}$ is a Carathéodory function.

$\left(A_{2}\right) \quad$ There exists an $N$-function $P$ with $P \ll M$, a function $c \in E_{\bar{M}}(\Omega)$, and positive constants $k_{1}, k_{2}, k_{3}, k_{4}$ such that

$$
|a(x, s, \zeta)| \leq c(x)+k_{1} \bar{P}^{-1} M\left(k_{2}|s|\right)+k_{3} \bar{M}^{-1} M\left(k_{4}|\zeta|\right)
$$

for a.e. $x$ in $\Omega$ and for all $s \in \mathbb{R}, \zeta \in \mathbb{R}^{N}$.

$\left(A_{3}\right) \quad$ For a.e. $x$ in $\Omega, s \in \mathbb{R}$ and $\zeta, \zeta^{\prime}$ in $\mathbb{R}^{N}$ with $\zeta^{\prime} \neq \zeta$,

$$
\left[a(x, s, \zeta)-a\left(x, s, \zeta^{\prime}\right)\right]\left(\zeta-\zeta^{\prime}\right)>0 .
$$

$\left(A_{4}\right) \quad$ For a.e. $x$ in $\Omega$ and all $\zeta \in \mathbb{R}^{N}$,

$$
a(x, s, \zeta) \zeta \geq \alpha M(|\zeta| / \nu) .
$$

$\left(A_{5}\right) \quad$ For each $v \in K_{\psi} \cap L^{\infty}(\Omega)$ there exists a sequence $v_{j} \in K_{\psi} \cap$ $W_{0}^{1} E_{M}(\Omega) \cap L^{\infty}(\Omega)$ such that

$$
v_{j} \rightarrow v \quad \text { for the modular convergence. }
$$


Finally, we suppose that

$$
\begin{gathered}
\mu \in L^{1}(\Omega)+W^{-1} E_{\bar{M}}(\Omega), \\
\phi \in C^{0}\left(\mathbb{R}, \mathbb{R}^{N}\right), \\
K_{\psi} \cap L^{\infty}(\Omega) \neq \emptyset .
\end{gathered}
$$

REMARK 3.1. Condition $\left(A_{5}\right)$ holds if one of the following conditions is satisfied:

(a) There exists $\bar{\psi} \in K_{\psi}$ such that $\psi-\bar{\psi}$ is continuous in $\Omega$ (see [14, Proposition 9]).

(b) $\psi \in W_{0}^{1} E_{M}(\Omega)$ (see [14, Proposition 10]).

(c) The $N$-function $M$ satisfies the $\Delta_{2}$-condition near infinity.

(d) $\psi=-\infty$ (i.e., $K_{\psi}=W_{0}^{1} L_{M}(\Omega)$ ).

3.2. Principal result. Since $\mu \in L^{1}(\Omega)+W^{-1} E_{\bar{M}}(\Omega)$, it can be written as follows:

$$
\mu=f-\operatorname{div} F \quad \text { with } f \in L^{1}(\Omega), F \in\left(E_{\bar{M}}(\Omega)\right)^{N} .
$$

We consider the following unilateral problem:

$$
\left\{\begin{array}{r}
u \in \mathcal{T}_{0}^{1, M}(\Omega), \quad u \geq \psi \text { a.e. in } \Omega \\
\int_{\Omega} a(x, u, \nabla u) \nabla T_{k}(u-v) d x+\int_{\Omega} \phi(u) \nabla T_{k}(u-v) d x \\
\leq \int_{\Omega} f T_{k}(u-v) d x+\int_{\Omega} F \nabla T_{k}(u-v) d x, \\
\forall v \in K_{\psi} \cap L^{\infty}(\Omega), \forall k>0 .
\end{array}\right.
$$

We prove the following existence result.

TheOREM 3.1. Under the assumptions $\left(A_{1}\right)-\left(A_{5}\right)$ and (3.1)-(3.3), there exists at least one solution of the problem (3.4).

REMARK 3.2. In the previous result, we cannot replace $K_{\psi} \cap L^{\infty}(\Omega)$ by just $K_{\psi}$, since in general the integral $\int_{\Omega} \phi(u) \nabla T_{k}(u-v) d x$ may not have a meaning.

REMARK 3.3. If we take $M(t)=|t|^{p}$ in the previous statement, we obtain an existence result in the classical Sobolev spaces (which seems to be new).

REMARK 3.4. The statement of Theorem 3.1 holds when $\mu \in L^{1}(\Omega)+$ $W^{-1} L_{\bar{M}}(\Omega)$.

It suffices to approximate $\mu=f-\operatorname{div} F$ with $F=\left(F_{1}, \ldots, F_{N}\right) \in$ $\left(L_{\bar{M}}(\Omega)\right)^{N}$ by $\mu_{n}=f_{n}-\operatorname{div} F_{n}$ where $f_{n}$ is a regular function such that $f_{n}$ strongly converges to $f$ in $L^{1}(\Omega)$ and $F_{n}=\left(T_{n}\left(F_{1}\right), \ldots, T_{n}\left(F_{N}\right)\right)$.

We write $\varepsilon(n, i, j)$ for any quantity such that

$$
\lim _{j \rightarrow \infty} \lim _{i \rightarrow \infty} \lim _{n \rightarrow \infty} \varepsilon(n, i, j)=0 .
$$


The notations $\varepsilon(n, j)$ etc. are defined similarly. Finally, we denote (for example) by $\varepsilon_{j}(n, i)$ a quantity that depends on $n, i, j$ and is such that

$$
\lim _{i \rightarrow \infty} \lim _{n \rightarrow \infty} \varepsilon_{j}(n, i)=0
$$

for any fixed value of $j$.

4. Proof of principal result. Without loss the generality we take $\nu=1$ in condition $\left(A_{4}\right)$. We fix a function $v_{0} \in K_{\psi} \cap W_{0}^{1} E_{M}(\Omega) \cap L^{\infty}(\Omega)$ (such a function exists by $\left(A_{5}\right)$ and (3.3)).

4.1. Approximate problem. We consider the sequence of approximate problems

$$
\left\{\begin{array}{l}
u_{n} \in K_{\psi}, \\
\left\langle A u_{n}, u_{n}-v\right\rangle+\int_{\Omega} \phi\left(T_{n}\left(u_{n}\right)\right) \nabla\left(u_{n}-v\right) d x \\
\leq \int_{\Omega} f_{n}\left(u_{n}-v\right) d x+\int_{\Omega} F \nabla\left(u_{n}-v\right) d x \quad \forall v \in K_{\psi} .
\end{array}\right.
$$

where $f_{n}$ is a regular function such that $f_{n}$ strongly converges to $f$ in $L^{1}(\Omega)$. This approximate problem has a solution by the classical result of [14].

4.2. Some intermediate results. Let us prove the following lemma which is needed below:

Lemma 4.1. Assume that $\left(A_{1}\right)-\left(A_{4}\right)$ are satisfied, and let $\left(z_{n}\right)_{n}$ be a sequence in $W_{0}^{1} L_{M}(\Omega)$ such that

(a) $z_{n} \rightarrow z$ in $W_{0}^{1} L_{M}(\Omega)$ for $\sigma\left(\prod L_{M}(\Omega), \prod E_{\bar{M}}(\Omega)\right)$;

(b) $\left(a\left(x, z_{n}, \nabla z_{n}\right)\right)_{n}$ is bounded in $\left(L_{\bar{M}}(\Omega)\right)^{N}$;

(c) $\int_{\Omega}\left[a\left(x, z_{n}, \nabla z_{n}\right)-a\left(x, z_{n}, \nabla z \chi_{s}\right)\right]\left[\nabla z_{n}-\nabla z \chi_{s}\right] d x \rightarrow 0$ as $n, s \rightarrow \infty$ (where $\chi_{s}$ is the characteristic function of $\Omega_{s}=\{x \in \Omega:|\nabla z| \leq s\}$ ).

Then

$$
M\left(\left|\nabla z_{n}\right|\right) \rightarrow M(|\nabla z|) \quad \text { in } L^{1}(\Omega) .
$$

REMARK 4.1. Condition (b) is not necessary if $M$ satisfies the $\Delta_{2^{-}}$ condition.

Indeed, (a) implies that $\left(z_{n}\right)_{n}$ is bounded in $W_{0}^{1} L_{M}(\Omega)$, hence there exist two positive constants $\lambda, C$ such that

$$
\int_{\Omega} M\left(\lambda\left|\nabla z_{n}\right|\right) d x \leq C .
$$

On the other hand, let $Q$ be an $N$-function such that $M \ll Q$ and the continuous embedding $W_{0}^{1} L_{M}(\Omega) \subset E_{Q}(\Omega)$ holds (see [13]). Let $\varepsilon>0$. Then there exists $C_{\varepsilon}>0$, as in [7], such that

$$
|a(x, s, \zeta)| \leq c(x)+C_{\varepsilon}+k_{1} \bar{M}^{-1} Q(\varepsilon|s|)+k_{3} \bar{M}^{-1} M(\varepsilon|\zeta|)
$$


for a.e. $x \in \Omega$ and all $(s, \zeta) \in \mathbb{R} \times \mathbb{R}^{N}$. From (4.2) and (4.3) we deduce that $\left(a\left(x, z_{n}, \nabla z_{n}\right)\right)_{n}$ is bounded in $\left(L_{\bar{M}}(\Omega)\right)^{N}$.

Proof of Lemma 4.1. Fix $r>0$ and let $s>r$. We have

$$
\begin{aligned}
0 & \leq \int_{\Omega_{r}}\left[a\left(x, z_{n}, \nabla z_{n}\right)-a\left(x, z_{n}, \nabla z\right)\right]\left[\nabla z_{n}-\nabla z\right] d x \\
& \leq \int_{\Omega_{s}}\left[a\left(x, z_{n}, \nabla z_{n}\right)-a\left(x, z_{n}, \nabla z\right)\right]\left[\nabla z_{n}-\nabla z\right] d x \\
& =\int_{\Omega_{s}}\left[a\left(x, z_{n}, \nabla z_{n}\right)-a\left(x, z_{n}, \nabla z \chi_{s}\right)\right]\left[\nabla z_{n}-\nabla z \chi_{s}\right] d x \\
& \leq \int_{\Omega}\left[a\left(x, z_{n}, \nabla z_{n}\right)-a\left(x, z_{n}, \nabla z \chi_{s}\right)\right]\left[\nabla z_{n}-\nabla z \chi_{s}\right] d x .
\end{aligned}
$$

Together with (c) this implies

$$
\lim _{n \rightarrow \infty} \int_{\Omega_{r}}\left[a\left(x, z_{n}, \nabla z_{n}\right)-a\left(x, z_{n}, \nabla z\right)\right]\left[\nabla z_{n}-\nabla z\right] d x=0 .
$$

So, as in [13],

$$
\nabla z_{n} \rightarrow \nabla z \quad \text { a.e. in } \Omega .
$$

On the one hand, we have

$$
\begin{aligned}
\int_{\Omega} a\left(x, z_{n}, \nabla z_{n}\right) \nabla z_{n} d x= & \int_{\Omega}\left[a\left(x, z_{n}, \nabla z_{n}\right)-a\left(x, z_{n}, \nabla z \chi_{s}\right)\right] \\
& \times\left[\nabla z_{n}-\nabla z \chi_{s}\right] d x \\
& +\int_{\Omega} a\left(x, z_{n}, \nabla z \chi_{s}\right)\left(\nabla z_{n}-\nabla z \chi_{s}\right) d x \\
& +\int_{\Omega} a\left(x, z_{n}, \nabla z_{n}\right) \nabla z \chi_{s} d x
\end{aligned}
$$

Since $\left(a\left(x, z_{n}, \nabla z_{n}\right)\right)_{n}$ is bounded in $\left(L_{\bar{M}}(\Omega)\right)^{N}$, from (4.4), we obtain

$$
a\left(x, z_{n}, \nabla z_{n}\right) \rightarrow a(x, z, \nabla z) \quad \text { weakly in }\left(L_{\bar{M}}(\Omega)\right)^{N} \text { for } \sigma\left(\prod L_{\bar{M}}, \prod E_{M}\right) \text {. }
$$

Consequently,

$$
\int_{\Omega} a\left(x, z_{n}, \nabla z_{n}\right) \nabla z \chi_{s} d x \rightarrow \int_{\Omega} a(x, z, \nabla z) \nabla z \chi_{s} d x
$$

as $n \rightarrow \infty$. Letting also $s \rightarrow \infty$, we get

$$
\int_{\Omega} a(x, z, \nabla z) \nabla z \chi_{s} d x \rightarrow \int_{\Omega} a(x, z, \nabla z) \nabla z d x .
$$

On the other hand, it is easy to see that the second term on the right hand side of (4.5) tends to 0 as $n, s \rightarrow \infty$. 
Moreover, from (c), (4.6) and (4.7) we have

$$
\lim _{n \rightarrow \infty} \int_{\Omega} a\left(x, z_{n}, \nabla z_{n}\right) \nabla z_{n} d x=\int_{\Omega} a(x, z, \nabla z) \nabla z d x .
$$

Finally, using $\left(A_{4}\right)$, by Lemma 2.6 and Vitali's theorem, one obtains the assertion.

Proposition 4.1. Assume that $\left(A_{1}\right)-\left(A_{5}\right)$ and (3.1)-(3.3) hold and let $u_{n}$ be a solution of the approximate problem (4.1). Then for all $k>0$, there exists a constant $c(k)$ (which does not depend on $n$ ) such that

$$
\int_{\Omega} M\left(\left|\nabla T_{k}\left(u_{n}\right)\right|\right) \leq c(k) .
$$

Proof. Let $k>0$. Taking $u_{n}-T_{k}\left(u_{n}-v_{0}\right)$ as a test function in (4.1), we obtain, for $n$ large enough,

$$
\begin{aligned}
\int_{\Omega} a\left(x, u_{n}, \nabla u_{n}\right) \nabla T_{k}\left(u_{n}\right. & \left.-v_{0}\right) d x+\int_{\Omega} \phi\left(u_{n}\right) \nabla T_{k}\left(u_{n}-v_{0}\right) d x \\
& \leq \int_{\Omega} f_{n} T_{k}\left(u_{n}-v_{0}\right) d x+\int_{\Omega} F \nabla T_{k}\left(u_{n}-v_{0}\right) d x .
\end{aligned}
$$

Since $\nabla T_{k}\left(u_{n}-v_{0}\right)$ is identically zero on the set where $\left|u_{n}-v_{0}\right|>k$, we can write

$$
\begin{aligned}
& \int_{\Omega} a\left(x, u_{n}, \nabla u_{n}\right) \nabla T_{k}\left(u_{n}-v_{0}\right) d x \\
& \leq \int_{\left\{\left|u_{n}-v_{0}\right| \leq k\right\}}\left|\phi\left(T_{k+\left\|v_{0}\right\|_{\infty}}\left(u_{n}\right)\right)\right|\left|\nabla u_{n}\right| d x \\
& +\int_{\left\{\left|u_{n}-v_{0}\right| \leq k\right\}}\left|\phi\left(T_{k+\left\|v_{0}\right\|_{\infty}}\left(u_{n}\right)\right)\right|\left|\nabla v_{0}\right| d x \\
& +\int_{\Omega} f_{n} T_{k}\left(u_{n}-v_{0}\right) d x+\int_{\Omega} F \nabla T_{k}\left(u_{n}-v_{0}\right) d x .
\end{aligned}
$$

Now observe that (for $0<c<1$ )

$$
\begin{aligned}
& \int_{\left\{\left|u_{n}-v_{0}\right| \leq k\right\}} a\left(x, u_{n}, \nabla u_{n}\right) \nabla u_{n} d x \leq c \int_{\left\{\left|u_{n}-v_{0}\right| \leq k\right\}} a\left(x, u_{n}, \nabla u_{n}\right) \frac{\nabla v_{0}}{c} d x
\end{aligned}
$$

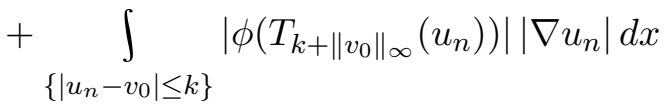

$$
\begin{aligned}
& +\int_{\left\{\left|u_{n}-v_{0}\right| \leq k\right\}}\left|\phi\left(T_{k+\left\|v_{0}\right\|_{\infty}}\left(u_{n}\right)\right)\right|\left|\nabla v_{0}\right| d x+\int_{\Omega} f_{n} T_{k}\left(u_{n}-v_{0}\right) d x \\
& +\int_{\Omega} F \nabla T_{k}\left(u_{n}-v_{0}\right) d x \text {. }
\end{aligned}
$$


By using $\left(A_{3}\right)$, we get

$$
\begin{aligned}
c \int_{\left\{\left|u_{n}-v_{0}\right| \leq k\right\}} a\left(x, u_{n}, \nabla u_{n}\right) \frac{\nabla v_{0}}{c} d x \\
\leq c \\
\quad\left\{\int_{\left\{\left|u_{n}-v_{0}\right| \leq k\right\}} a\left(x, u_{n}, \nabla u_{n}\right) \nabla u_{n} d x\right. \\
\left.-\int_{\left\{\left|u_{n}-v_{0}\right| \leq k\right\}} a\left(x, u_{n}, \frac{\nabla v_{0}}{c}\right)\left(\nabla u_{n}-\frac{\nabla v_{0}}{c}\right) d x\right\},
\end{aligned}
$$

which yields, thanks to (4.8),

$$
\begin{aligned}
& (1-c) \int_{\left\{\left|u_{n}-v_{0}\right| \leq k\right\}} a\left(x, u_{n}, \nabla u_{n}\right) \nabla u_{n} d x \\
& \leq \int_{\left\{\left|u_{n}-v_{0}\right| \leq k\right\}}\left|\phi\left(T_{k+\left\|v_{0}\right\|_{\infty}}\left(u_{n}\right)\right)\right|\left|\nabla u_{n}\right| d x
\end{aligned}
$$

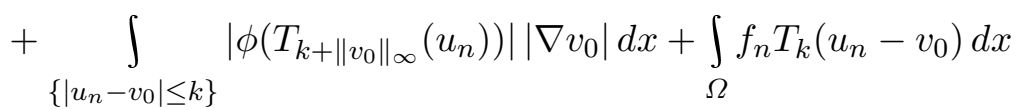

$$
\begin{aligned}
& +\int_{\Omega} F \nabla T_{k}\left(u_{n}-v_{0}\right) d x-c \int_{\left\{\left|u_{n}-v_{0}\right| \leq k\right\}} a\left(x, u_{n}, \frac{\nabla v_{0}}{c}\right)\left(\nabla u_{n}-\frac{\nabla v_{0}}{c}\right) d x .
\end{aligned}
$$

Since $\frac{\nabla v_{0}}{c} \in\left(E_{M}(\Omega)\right)^{N}$, using $\left(A_{2}\right)$ and the Young inequality, we have

$$
\begin{aligned}
&(1-c) \int_{\left\{\left|u_{n}-v_{0}\right| \leq k\right\}} a\left(x, u_{n}, \nabla u_{n}\right) \nabla u_{n} d x \\
& \leq \frac{\alpha(1-c)}{2} \int_{\left\{\left|u_{n}-v_{0}\right| \leq k\right\}} M\left(\left|\nabla u_{n}\right|\right) d x+c_{3}(k)
\end{aligned}
$$

where $c_{3}(k)$ is a positive constant which depends only on $k$.

Using also $\left(A_{4}\right)$ we obtain

$$
\frac{\alpha(1-c)}{2} \int_{\left\{\left|u_{n}-v_{0}\right| \leq k\right\}} M\left(\left|\nabla u_{n}\right|\right) d x \leq c_{3}(k) .
$$

Moreover, from $\left\{\left|u_{n}\right| \leq k\right\} \subset\left\{\left|u_{n}-v_{0}\right| \leq k+\left\|v_{0}\right\|_{\infty}\right\}$, we conclude that

$$
\int_{\Omega} M\left(\left|\nabla T_{k}\left(u_{n}\right)\right|\right) d x \leq c_{4}(k) .
$$

Proposition 4.2. Assume that $\left(A_{1}\right)-\left(A_{5}\right)$ and (3.1)-(3.3) hold, and let $u_{n}$ be a solution of the approximate problem (4.1). Then there exists a measurable function $u$ such that for all $k>0$ we have (for a subsequence still denoted by $u_{n}$ ), 
(i) $u_{n} \rightarrow u$ a.e. in $\Omega$,

(ii) $T_{k}\left(u_{n}\right) \rightarrow T_{k}(u)$ weakly in $W_{0}^{1} L_{M}(\Omega)$ for $\sigma\left(\prod L_{M}, \prod E_{\bar{M}}\right)$, $T_{k}\left(u_{n}\right) \rightarrow T_{k}(u)$ strongly in $E_{M}(\Omega)$ and a.e. in $\Omega$.

Before proving this proposition, we begin with the following estimate:

LEMMA 4.2. If $u_{n}$ is a solution of (4.1), then for $k>h>\left\|v_{0}\right\|_{\infty}$, we have

$$
\int_{\Omega} M\left(\left|\nabla T_{k}\left(u_{n}-T_{h}\left(u_{n}\right)\right)\right|\right) d x \leq k C,
$$

where $C$ is a constant that does not depend of $n, k$ and $h$.

Proof. By Proposition 4.1, there exists some $v_{k} \in W_{0}^{1} L_{M}(\Omega)$ such that

$$
\begin{aligned}
& T_{k}\left(u_{n}\right) \rightarrow v_{k} \quad \text { weakly in } W_{0}^{1} L_{M}(\Omega) \text { for } \sigma\left(\prod L_{M}, \prod E_{\bar{M}}\right), \\
& T_{k}\left(u_{n}\right) \rightarrow v_{k} \quad \text { strongly in } E_{M}(\Omega) \text { and a.e. in } \Omega .
\end{aligned}
$$

On the other hand, let $k>h \geq\left\|v_{0}\right\|_{\infty}$. By using $v=u_{n}-T_{k}\left(u_{n}-T_{h}\left(u_{n}\right)\right)$ as a test function in (4.1) we obtain

$$
\begin{aligned}
\int_{\Omega} a\left(x, u_{n}, \nabla u_{n}\right) \nabla T_{k}\left(u_{n}-T_{h}\left(u_{n}\right)\right) d x+\int_{\Omega} \phi\left(T_{n}\left(u_{n}\right)\right) \nabla T_{k}\left(u_{n}-T_{h}\left(u_{n}\right)\right) d x \\
\leq \int_{\Omega} f_{n} T_{k}\left(u_{n}-T_{h}\left(u_{n}\right)\right) d x+\int_{\Omega} F \nabla T_{k}\left(u_{n}-T_{h}\left(u_{n}\right)\right) d x .
\end{aligned}
$$

The second term on the left hand side vanishes for $n$ large enough. Indeed, by Lemma 2.7 ,

$$
\begin{aligned}
\int_{\Omega} \phi\left(T_{n}\left(u_{n}\right)\right) \nabla T_{k}\left(u_{n}-T_{h}\left(u_{n}\right)\right) d x & =\int_{\Omega} \phi\left(u_{n}\right) \nabla T_{k}\left(u_{n}-T_{h}\left(u_{n}\right)\right) d x \\
& =\int_{\Omega} \operatorname{div}\left[\int_{0}^{u_{n}} \phi(s) \chi_{\{h \leq|s| \leq k+h\}} d s\right] d x=0
\end{aligned}
$$

(since $\int_{0}^{u_{n}} \phi(s) \chi_{\{h \leq|s| \leq k+h\}} d s$ lies in $W_{0}^{1} L_{M}(\Omega)$ ). Thus,

$$
\begin{aligned}
\int_{\Omega} a\left(x, u_{n}, \nabla u_{n}\right) & \nabla T_{k}\left(u_{n}-T_{h}\left(u_{n}\right)\right) d x \\
\leq & \int_{\Omega} f_{n} T_{k}\left(u_{n}-T_{h}\left(u_{n}\right)\right) d x+\int_{\Omega} F \nabla T_{k}\left(u_{n}-T_{h}\left(u_{n}\right)\right) d x
\end{aligned}
$$

which yields the conclusion by using $\left(A_{4}\right)$ and Young's inequality.

Proof of Proposition 4.2. (i) We prove that $u_{n}$ converges to some function $u$ in measure (and hence a.e. by passing to a suitable subsequence). We shall show that $u_{n}$ is a Cauchy sequence in measure. 
Let $k>h>\left\|v_{0}\right\|_{\infty}$ be large enough. Thanks to Lemma 5.7 of [13], there exist two positive constants $C_{7}$ and $C_{8}$ independent of $k$ and $h$ such that

$$
\int_{\Omega} M\left(C_{7}\left|T_{k}\left(u_{n}-T_{h}\left(u_{n}\right)\right)\right|\right) d x \leq C_{8} \int_{\Omega} M\left(\left|\nabla T_{k}\left(u_{n}-T_{h}\left(u_{n}\right)\right)\right|\right) d x .
$$

By Lemma 4.2 this yields

$$
\begin{aligned}
M\left(C_{7} k\right) \operatorname{meas}\left\{\left|u_{n}-T_{h}\left(u_{n}\right)\right|>k\right\} & \\
& =\int_{\left\{\left|u_{n}-T_{h}\left(u_{n}\right)\right|>k\right\}} M\left(C_{7}\left|T_{k}\left(u_{n}-T_{h}\left(u_{n}\right)\right)\right|\right) d x \\
& \leq C_{8} \int_{\Omega} M\left(\left|\nabla T_{k}\left(u_{n}-T_{h}\left(u_{n}\right)\right)\right|\right) d x \leq k C_{9} .
\end{aligned}
$$

Consequently,

$$
\operatorname{meas}\left(\left\{\left|u_{n}-T_{h}\left(u_{n}\right)\right|>k\right\}\right) \leq \frac{k C_{9}}{M\left(k C_{7}\right)}
$$

for all $n$ and all $k>h>\left\|v_{0}\right\|_{\infty}$. Hence,

$\operatorname{meas}\left\{\left|u_{n}\right|>k\right\} \leq \operatorname{meas}\left\{\left|u_{n}-T_{h}\left(u_{n}\right)\right|>k-h\right\} \leq \frac{(k-h) C_{9}}{M\left((k-h) C_{7}\right)} \quad$ for all $n$.

Therefore, since $t / M(t) \rightarrow 0$ as $t \rightarrow \infty$, we obtain

$$
\text { meas }\left\{\left|u_{n}\right|>k\right\} \rightarrow 0 \quad \text { uniformly in } n \text { as } k \rightarrow \infty \text {. }
$$

Now, for $\lambda>0$, we have

$$
\begin{aligned}
\operatorname{meas}\left(\left\{\left|u_{n}-u_{m}\right|>\lambda\right\}\right) \leq & \operatorname{meas}\left(\left\{\left|u_{n}\right|>k\right\}\right)+\operatorname{meas}\left(\left\{\left|u_{m}\right|>k\right\}\right) \\
& +\operatorname{meas}\left(\left\{\left|T_{k}\left(u_{n}\right)-T_{k}\left(u_{m}\right)\right|>\lambda\right\}\right) .
\end{aligned}
$$

From (4.11), we can assume that $T_{k}\left(u_{n}\right)$ is a Cauchy sequence in measure in $\Omega$.

Let $\varepsilon>0$. By (4.12), (4.13) and the fact that $T_{k}\left(u_{n}\right)$ is a Cauchy sequence in measure, there exists some $k(\varepsilon)>0$ such that meas $\left(\left\{\left|u_{n}-u_{m}\right|>\lambda\right\}\right)<\varepsilon$ for all $n, m \geq n_{0}(k(\varepsilon), \lambda)$. This proves that $\left(u_{n}\right)_{n}$ is a Cauchy sequence in measure in $\Omega$, thus it converges almost everywhere to some measurable function $u$.

(ii) It suffices to combine assertion (i) and (4.11).

Proposition 4.3. Assume that $\left(A_{1}\right)-\left(A_{5}\right)$ and (3.1)-(3.3) hold and let $u_{n}$ be a solution of the approximate problem (4.1). Then for all $k>0$,

(i) $\left(a\left(x, T_{k}\left(u_{n}\right), \nabla T_{k}\left(u_{n}\right)\right)\right)_{n}$ is bounded in $\left(L_{\bar{M}}(\Omega)\right)^{N}$,

(ii) $\nabla u_{n} \rightarrow \nabla u$ a.e. in $\Omega$.

Proof. (i) Let $w \in\left(E_{M}(\Omega)\right)^{N}$. By condition $\left(A_{3}\right)$ we have

$$
\left(a\left(x, u_{n}, \nabla u_{n}\right)-a\left(x, u_{n}, w\right)\right)\left(\nabla u_{n}-w\right) \geq 0 .
$$


Consequently,

$$
\begin{aligned}
\int_{\left\{\left|u_{n}\right| \leq k\right\}} a\left(x, u_{n}, \nabla u_{n}\right) w d x \leq & \int_{\left\{\left|u_{n}\right| \leq k\right\}} a\left(x, u_{n}, \nabla u_{n}\right) \nabla u_{n} d x \\
& +\int_{\left\{\left|u_{n}\right| \leq k\right\}} a\left(x, u_{n}, w\right)\left(w-\nabla u_{n}\right) d x .
\end{aligned}
$$

Combining (4.9) and (4.10) and using the fact that $\left\{\left|u_{n}\right| \leq k\right\} \subset\left\{\left|u_{n}-v_{0}\right| \leq\right.$ $\left.k+\left\|v_{0}\right\|_{\infty}\right\}$, we get

$$
\int_{\Omega} a\left(x, T_{k}\left(u_{n}\right), \nabla T_{k}\left(u_{n}\right)\right) \nabla T_{k}\left(u_{n}\right) d x \leq C_{10},
$$

where $C_{10}$ is a positive constant.

On the other hand, by $\left(A_{2}\right)$ we have

$$
\left|a\left(x, T_{k}\left(u_{n}\right), w\right)\right| \leq c(x)+k_{1} \bar{P}^{-1} M\left(k_{2}\left|T_{k}(u)\right|\right)+k_{3} \bar{M}^{-1} M\left(k_{4}|w|\right) .
$$

Therefore,

$$
\int_{\Omega} \bar{M}\left(\frac{a\left(x, T_{k}\left(u_{n}\right), w\right)}{\lambda}\right) d x \leq \int_{\Omega} \bar{M}\left(\frac{c(x)}{\lambda}\right)+\int_{\Omega} \frac{k_{3}}{\lambda} M\left(k_{4}|w|\right)+C_{11} \leq C_{12}
$$

when $\lambda>0$ is large enough. Hence $\left\{a\left(x, T_{k}\left(u_{n}\right), w\right)\right\}$ is bounded in $\left(L_{\bar{M}}(\Omega)\right)^{N}$. This implies that the second term on the right in (4.14) is also bounded. By the theorem of Banach-Steinhaus, the sequence $\left(a\left(x, T_{k}\left(u_{n}\right), \nabla T_{k}\left(u_{n}\right)\right)\right)_{n}$ remains bounded in $\left(L_{\bar{M}}(\Omega)\right)^{N}$.

(ii) Let $k>\left\|v_{0}\right\|_{\infty}$. By $\left(A_{5}\right)$ there exists a sequence $v_{j} \in K_{\psi} \cap W_{0}^{1} E_{M}(\Omega)$ $\cap L^{\infty}(\Omega)$ such that

$$
v_{j} \rightarrow T_{k}(u) \quad(\bmod ) \text { in } W_{0}^{1} L_{M}(\Omega) .
$$

Fix $r$ and let $s>r$. Let $\Omega_{r}=\left\{x \in \Omega:\left|\nabla T_{k}(u(x))\right| \leq r\right\}$ and denote by $\chi_{r}$ the characteristic function of $\Omega_{r}$. Consider the expression

$$
\begin{aligned}
I_{n, r}=\int_{\Omega_{r}}\left\{\left[a\left(x, T_{k}\left(u_{n}\right), \nabla T_{k}\left(u_{n}\right)\right)-a\left(x, T_{k}\left(u_{n}\right), \nabla T_{k}(u)\right)\right]\right. & \\
& \left.\times\left[\nabla T_{k}\left(u_{n}\right)-\nabla T_{k}(u)\right]\right\}^{\theta} d x
\end{aligned}
$$

where $0<\theta<1$. Let $A_{n}$ be the expression in braces above. Then for any $0<\eta<1$,

$$
I_{n, r}=\int_{\Omega_{r} \cap\left\{\left|T_{k}\left(u_{n}\right)-T_{k}\left(v_{j}\right)\right| \leq \eta\right\}} A_{n}^{\theta} d x+\int_{\Omega_{r} \cap\left\{\left|T_{k}\left(u_{n}\right)-T_{k}\left(v_{j}\right)\right|>\eta\right\}} A_{n}^{\theta} d x .
$$

Since $\left(a\left(x, T_{k}\left(u_{n}\right), \nabla T_{k}\left(u_{n}\right)\right)\right)_{n}$ is bounded in $\left(L_{\bar{M}}(\Omega)\right)^{N}$, while $\nabla T_{k}\left(u_{n}\right)$ is bounded in $\left(L_{M}(\Omega)\right)^{N}$, by applying Hölder's inequality, we obtain

$$
\begin{aligned}
I_{n, r} \leq & c_{1}\left(\int_{\Omega_{r} \cap\left\{\left|T_{k}\left(u_{n}\right)-T_{k}\left(v_{j}\right)\right| \leq \eta\right\}} A_{n} d x\right)^{\theta} \\
& +c_{2} \operatorname{meas}\left\{x:\left|T_{k}\left(u_{n}\right)-T_{k}\left(v_{j}\right)\right|>\eta\right\}^{1-\theta} .
\end{aligned}
$$


Now observe that

$$
\begin{gathered}
\text { 17) } \int_{\Omega_{r} \cap\left\{\left|T_{k}\left(u_{n}\right)-T_{k}\left(v_{j}\right)\right| \leq \eta\right\}} A_{n} d x \\
\leq \int_{\left\{\left|T_{k}\left(u_{n}\right)-T_{k}\left(v_{j}\right)\right| \leq \eta\right\}}\left[a\left(x, T_{k}\left(u_{n}\right), \nabla T_{k}\left(u_{n}\right)\right)-a\left(x, T_{k}\left(u_{n}\right), \nabla T_{k}(u) \chi_{s}\right)\right] \\
=\quad \int_{\left\{\left|T_{k}\left(u_{n}\right)-T_{k}\left(v_{j}\right)\right| \leq \eta\right\}} a\left(x, T_{k}\left(u_{n}\right), \nabla T_{k}\left(u_{n}\right)\right)\left(\nabla T_{k}\left(u_{n}\right)-\nabla T_{k}(u) \chi_{s}\right] d x \\
-\quad \int_{\left\{\left|T_{k}\left(u_{n}\right)-T_{k}\left(v_{j}\right)\right| \leq \eta\right\}} a\left(x, T_{k}\left(u_{n}\right), \nabla T_{k}(u) \chi_{s}\right)\left(\nabla T_{k}\left(u_{n}\right)-\nabla T_{k}(u) \chi_{s}\right) d x \\
+\quad \int_{\left\{\left|T_{k}\left(u_{n}\right)-T_{k}\left(v_{j}\right)\right| \leq \eta\right\}} a\left(x, T_{k}\left(u_{n}\right), \nabla T_{k}\left(u_{n}\right)\right) \nabla T_{k}\left(v_{j}\right) d x \\
-\quad a\left(x, T_{k}\left(u_{n}\right), \nabla T_{k}\left(u_{n}\right)\right) \nabla T_{k}(u) \chi_{s} d x,
\end{gathered}
$$

and since $\left(a\left(x, T_{k}\left(u_{n}\right), \nabla T_{k}\left(u_{n}\right)\right)\right)_{n}$ is bounded in $\left(L_{\bar{M}}(\Omega)\right)^{N}$ by Proposition 4.3(i), there exists $\varrho_{k} \in\left(L_{\bar{M}}(\Omega)\right)^{N}$ such that

$$
\begin{aligned}
a\left(x, T_{k}\left(u_{n}\right), \nabla T_{k}\left(u_{n}\right)\right) & \rightarrow \varrho_{k} \\
\text { weakly in }\left(L_{\bar{M}}(\Omega)\right)^{N} & \text { for } \sigma\left(\prod L_{M}, \prod E_{\bar{M}}\right)
\end{aligned}
$$

as $n \rightarrow \infty$. Letting $n$ tend to infinity, we obtain

$$
\begin{gathered}
\int_{\left\{\left|T_{k}\left(u_{n}\right)-T_{k}\left(v_{j}\right)\right| \leq \eta\right\}} a\left(x, T_{k}\left(u_{n}\right), \nabla T_{k}\left(u_{n}\right)\right) \nabla T_{k}(u) \chi_{s} d x \\
=\int_{\left\{\left|T_{k}(u)-T_{k}\left(v_{j}\right)\right| \leq \eta\right\}} \varrho_{k} \nabla T_{k}(u) \chi_{s} d x+\varepsilon(n), \\
\int_{\left\{\left|T_{k}\left(u_{n}\right)-T_{k}\left(v_{j}\right)\right| \leq \eta\right\}} a\left(x, T_{k}\left(u_{n}\right), \nabla T_{k}\left(u_{n}\right)\right) \nabla T_{k}\left(v_{j}\right) d x \\
=\int_{\left\{\left|T_{k}(u)-T_{k}\left(v_{j}\right)\right| \leq \eta\right\}} \varrho_{k} \nabla T_{k}\left(v_{j}\right) d x+\varepsilon(n),
\end{gathered}
$$

and hence by letting $j \rightarrow \infty$ and using (4.15),

$$
\begin{aligned}
\int_{\left\{\left|T_{k}\left(u_{n}\right)-T_{k}\left(v_{j}\right)\right| \leq \eta\right\}} a\left(x, T_{k}\left(u_{n}\right), \nabla T_{k}\left(u_{n}\right)\right) \nabla T_{k}(u) \chi_{s} d x \\
\quad=\int_{\Omega} \varrho_{k} \nabla T_{k}(u) \chi_{s} d x+\varepsilon(n, j),
\end{aligned}
$$


and

$$
\begin{gathered}
\int_{\left\{\left|T_{k}\left(u_{n}\right)-T_{k}\left(v_{j}\right)\right| \leq \eta\right\}} a\left(x, T_{k}\left(u_{n}\right), \nabla T_{k}\left(u_{n}\right)\right) \nabla T_{k}\left(v_{j}\right) d x \\
=\int_{\Omega} \varrho_{k} \nabla T_{k}(u) d x+\varepsilon(n, j) .
\end{gathered}
$$

Starting with the second term on the right hand side of (4.17), we have, by letting $n \rightarrow \infty$,

$$
\int_{\left\{\left|T_{k}\left(u_{n}\right)-T_{k}\left(v_{j}\right)\right| \leq \eta\right\}} a\left(x, T_{k}\left(u_{n}\right), \nabla T_{k}(u) \chi_{s}\right)\left[\nabla T_{k}\left(u_{n}\right)-\nabla T_{k}(u) \chi_{s}\right] d x=\varepsilon(n)
$$

since

$$
\begin{aligned}
a\left(x, T_{k}\left(u_{n}\right), \nabla T_{k}(u) \chi_{s}\right) \chi_{\left\{\left|T_{k}\left(u_{n}\right)-T_{k}\left(v_{j}\right)\right| \leq \eta\right\}} & \\
& \rightarrow a\left(x, T_{k}(u), \nabla T_{k}(u) \chi_{s}\right) \chi_{\left\{\left|T_{k}(u)-T_{k}\left(v_{j}\right)\right| \leq \eta\right\}}
\end{aligned}
$$

strongly in $\left(E_{\bar{M}}(\Omega)\right)^{N}$ by using Lemma 2.3 while $\nabla T_{k}\left(u_{n}\right) \rightarrow \nabla T_{k}\left(u_{n}\right)$ weakly in $\left(L_{M}(\Omega)\right)^{N}$ by (4.11) and Proposition 4.3(ii).

We now study the first term on the right hand side of (4.17). By using the test function $u_{n}-T_{\eta}\left(u_{n}-T_{k}\left(v_{j}\right)\right)$ in (4.1), we get

$$
\begin{array}{r}
\int_{\Omega} a\left(x, u_{n}, \nabla u_{n}\right) \nabla T_{\eta}\left(u_{n}-T_{k}\left(v_{j}\right)\right) d x+\int_{\Omega} \phi\left(u_{n}\right) \nabla T_{\eta}\left(u_{n}-T_{k}\left(v_{j}\right)\right) d x \\
\leq \int_{\Omega} f_{n} T_{\eta}\left(u_{n}-T_{k}\left(v_{j}\right)\right) d x+\int_{\Omega} F \nabla T_{\eta}\left(u_{n}-T_{k}\left(v_{j}\right)\right) d x .
\end{array}
$$

Splitting the first integral on the left hand side into the regions where $\left|u_{n}\right| \leq k$ and $\left|u_{n}\right|>k$, we can write

$$
\begin{aligned}
\int_{\Omega} a\left(x, u_{n}, \nabla u_{n}\right) \nabla T_{\eta}\left(u_{n}-T_{k}\left(v_{j}\right)\right) d x & \\
= & \int_{\left\{\left|u_{n}\right| \leq k\right\}} a\left(x, T_{k}\left(u_{n}\right), \nabla T_{k}\left(u_{n}\right)\right) \nabla T_{\eta}\left(T_{k}\left(u_{n}\right)-T_{k}\left(v_{j}\right)\right) d x \\
& +\int_{\left\{\left|u_{n}\right|>k\right\}} a\left(x, u_{n}, \nabla u_{n}\right) \nabla T_{\eta}\left(u_{n}-T_{k}\left(v_{j}\right)\right) d x,
\end{aligned}
$$

which implies, by using $\left(A_{4}\right)$,

$$
\begin{aligned}
\int_{\Omega} a\left(x, u_{n}, \nabla u_{n}\right) \nabla T_{\eta}\left(u_{n}-T_{k}\left(v_{j}\right)\right) d x \\
\geq \int_{\Omega} a\left(x, T_{k}\left(u_{n}\right), \nabla T_{k}\left(u_{n}\right)\right) \nabla T_{\eta}\left(T_{k}\left(u_{n}\right)-T_{k}\left(v_{j}\right)\right) d x \\
\quad-\int_{\left\{\left|u_{n}\right|>k\right\}}\left|a\left(x, T_{k+1}\left(u_{n}\right), \nabla T_{k+1}\left(u_{n}\right)\right)\right|\left|\nabla v_{j}\right| d x .
\end{aligned}
$$


Combining (4.22) and (4.23), we deduce

$$
\begin{aligned}
\int_{\Omega} a\left(x, T_{k}\left(u_{n}\right), \nabla T_{k}\left(u_{n}\right)\right) & \nabla T_{\eta}\left(T_{k}\left(u_{n}\right)-T_{k}\left(v_{j}\right)\right) d x \\
& +\int_{\Omega} \phi\left(u_{n}\right) \nabla T_{\eta}\left(u_{n}-T_{k}\left(v_{j}\right)\right) d x \\
\leq & \int_{\left\{\left|u_{n}\right|>k\right\}}\left|a\left(x, T_{k+1}\left(u_{n}\right), \nabla T_{k+1}\left(u_{n}\right)\right)\right|\left|\nabla v_{j}\right| d x \\
& +\int_{\Omega} F \nabla T_{\eta}\left(u_{n}-T_{k}\left(v_{j}\right)\right) d x+c_{1} \eta .
\end{aligned}
$$

Using the boundedness of $\left\{\left|a\left(x, T_{k+1}\left(u_{n}\right), \nabla T_{k+1}\left(u_{n}\right)\right)\right|\right\}_{n}$ in $L_{\bar{M}}(\Omega)$ and reasoning as above, it is easy to see that

$$
\int_{\left\{\left|u_{n}\right|>k\right\}}\left|a\left(x, T_{k+1}\left(u_{n}\right), \nabla T_{k+1}\left(u_{n}\right)\right)\right|\left|\nabla v_{j}\right| d x=\int_{\{|u|>k\}} h_{k}\left|\nabla v_{j}\right| d x,
$$

where $h_{k}$ is some function in $L_{\bar{M}}(\Omega)$ such that

$$
\left|a\left(x, T_{k+1}\left(u_{n}\right), \nabla T_{k+1}\left(u_{n}\right)\right)\right| \rightarrow h_{k} \quad \text { for } \sigma\left(L_{\bar{M}}(\Omega), E_{M}(\Omega)\right) \text { as } n \rightarrow \infty .
$$

Moreover, by (4.15) and the fact that $h_{k} \chi_{\{|u|>k\}} \in L_{M}(\Omega)$, we get

$$
\int_{\left\{\left|u_{n}\right|>k\right\}}\left|a\left(x, T_{k+1}\left(u_{n}\right), \nabla T_{k+1}\left(u_{n}\right)\right)\right|\left|\nabla v_{j}\right| d x=\varepsilon(n, j) .
$$

Similarly, we have

$$
\begin{array}{r}
\int_{\Omega} \phi\left(u_{n}\right) \nabla T_{\eta}\left(u_{n}-T_{k}\left(v_{j}\right)\right) d x=\int_{\Omega} \phi(u) \nabla T_{\eta}\left(u-T_{k}(u)\right) d x+\varepsilon(n, j)=\varepsilon(n, j), \\
\int_{\Omega} F \nabla T_{\eta}\left(u_{n}-T_{k}\left(v_{j}\right)\right) d x=\int_{\left\{\left|u-T_{k}(u)\right| \leq \eta,|u|>k\right\}} F \nabla T_{\eta}\left(u-T_{k}(u)\right) d x+\varepsilon(n, j) \\
\leq c_{3}\left\|F \chi_{\left\{\left|u-T_{k}(u)\right| \leq \eta,|u|>k\right\}}\right\|_{\bar{M}}\left\|\nabla T_{1}\left(u-T_{k}(u)\right)\right\|_{M}+\varepsilon(n, j) .
\end{array}
$$

Consequently, we deduce

$$
\begin{aligned}
& \int_{\Omega} a\left(x, T_{k}\left(u_{n}\right), \nabla T_{k}\left(u_{n}\right)\right) \nabla T_{\eta}\left(T_{k}\left(u_{n}\right)-T_{k}\left(v_{j}\right)\right) d x \\
& \quad \leq c_{3}\left\|F \chi_{\left\{\left|u-T_{k}(u)\right| \leq \eta,|u|>k\right\}}\right\|_{\bar{M}}\left\|\nabla T_{1}\left(u-T_{k}(u)\right)\right\|_{M}+\varepsilon(n, j) .
\end{aligned}
$$

Hence, from (4.17), (4.19), (4.20), (4.21) and (4.24), we get

$$
\begin{gathered}
\int_{\Omega_{r} \cap\left\{\left|T_{k}\left(u_{n}\right)-T_{k}\left(v_{j}\right)\right| \leq \eta\right\}} A_{n} d x \\
\leq c_{3}\left\|F \chi_{\left\{\left|u-T_{k}(u)\right| \leq \eta,|u|>k\right\}}\right\|_{\bar{M}}\left\|\nabla T_{1}\left(u-T_{k}(u)\right)\right\|_{M} \\
+\int_{\Omega \backslash \Omega_{s}} \varrho_{k} \nabla T_{k}(u) d x+C_{1} \eta+\varepsilon(n, j) .
\end{gathered}
$$


Finally, in virtue of (4.16) and (4.2), we deduce

$$
\begin{aligned}
I_{n, r} \leq & C_{4} \text { meas }\left\{x:\left|T_{k}\left(u_{n}\right)-T_{k}\left(v_{j}\right)\right|>\eta\right\}^{1-\theta}+\left\{C_{2} \int_{\Omega \backslash \Omega_{s}} \varrho_{k} \nabla T_{k}(u) d x\right. \\
& \left.+c_{3}\left\|F \chi_{\left\{\left|u-T_{k}(u)\right| \leq \eta,|u|>k\right\}}\right\|_{\bar{M}}\left\|\nabla T_{1}\left(u-T_{k}(u)\right)\right\|_{M}+C_{1} \eta+\varepsilon(n, j)\right\}^{\theta} .
\end{aligned}
$$

Consequently,

$$
\begin{aligned}
\limsup _{n \rightarrow \infty} & I_{n, r} \leq c_{4} \operatorname{meas}\left\{x:\left|T_{k}(u)-T_{k}\left(v_{j}\right)\right|>\eta\right\}^{1-\theta}+\left\{C_{2} \int_{\Omega \backslash \Omega_{s}} h_{k} \nabla T_{k}(u) d x\right. \\
& \left.+c_{3}\left\|F \chi_{\left\{\left|u-T_{k}(u)\right| \leq \eta,|u|>k\right\}}\right\|_{\bar{M}}\left\|\nabla T_{1}\left(u-T_{k}(u)\right)\right\|_{M}+C_{1} \eta+\varepsilon(n, j)\right\}^{\theta},
\end{aligned}
$$

in which we let successively $j \rightarrow \infty, s \rightarrow \infty$ and $\eta \rightarrow 0$ to obtain

$$
\limsup _{n \rightarrow \infty} I_{n, r}=0 \text {. }
$$

As in [13], this implies that there exists a subsequence also denoted by $u_{n}$ such that $\nabla u_{n} \rightarrow \nabla u$ a.e. in $\Omega$.

Proposition 4.4. Assume that $\left(A_{1}\right)-\left(A_{5}\right)$ and (3.1)-(3.3) hold and let $u_{n}$ be a solution of the approximate problem (4.1). Then for all $k>0$,

$$
M\left(\left|\nabla T_{k}\left(u_{n}\right)\right|\right) \rightarrow M\left(\left|\nabla T_{k}(u)\right|\right) \quad \text { in } L^{1}(\Omega) .
$$

Proof. We fix $k>\left\|v_{0}\right\|_{\infty}$. Then by $\left(A_{5}\right)$ there exists a sequence $v_{j} \in$ $K_{\psi} \cap W_{0}^{1} E_{M}(\Omega) \cap L^{\infty}(\Omega)$ which converges to $T_{k}(u)$ for the modular convergence in $W_{0}^{1} L_{M}(\Omega)$. We define

$$
\begin{aligned}
w_{n, j}^{h} & =T_{2 k}\left(u_{n}-T_{h}\left(u_{n}\right)+T_{k}\left(u_{n}\right)-T_{k}\left(v_{j}\right)\right), \\
w_{j}^{h} & =T_{2 k}\left(u-T_{h}(u)+T_{k}(u)-T_{k}\left(v_{j}\right)\right), \\
w^{h} & =T_{2 k}\left(u-T_{h}(u)\right),
\end{aligned}
$$

where $h>2 k$.

We choose $v=u_{n}-w_{n, j}^{h}$ as a test function in (4.1) to obtain

$$
\begin{aligned}
\int_{\Omega} a\left(x, u_{n}, \nabla u_{n}\right) \nabla w_{n, j}^{h} d x+\int_{\Omega} \phi\left(u_{n}\right) \nabla w_{n, j}^{h} d x & \\
\leq & \int_{\Omega} f_{n} w_{n, j}^{h} d x+\int_{\Omega} F \nabla w_{n, j}^{h} d x .
\end{aligned}
$$

By the strong convergence of $f_{n}$ and since $w_{n, j}^{h}$ converges to $w_{j}^{h}$ in the weak ${ }^{*}$ topology of $L^{\infty}(\Omega)$ as $n \rightarrow \infty$, we have

$$
\int_{\Omega} f_{n} w_{n, j}^{h} d x=\int_{\Omega} f w_{j}^{h} d x+\varepsilon(n)=\int_{\Omega} f w^{h} d x+\varepsilon(n, j) .
$$

The last passage is due to the fact that $w_{j}^{h}$ converges to zero in the weak* topology of $L^{\infty}(\Omega)$ as $j \rightarrow \infty$. 
Finally, letting $h \rightarrow \infty$, Lebesgue's theorem yields $\int_{\Omega} f w_{n, j}^{h} d x \rightarrow 0$, so

$$
\int_{\Omega} f_{n} w_{n, j}^{h} d x=\varepsilon(n, j, h) .
$$

We now study the first integral on the left hand side of (4.26):

$$
\begin{aligned}
\int_{\Omega} a\left(x, u_{n}, \nabla u_{n}\right) \nabla w_{n, j}^{h} d x \\
=\int_{\left\{\left|u_{n}\right| \leq k\right\}} a\left(x, T_{k}\left(u_{n}\right), \nabla T_{k}\left(u_{n}\right)\right)\left[\nabla T_{k}\left(u_{n}\right)-\nabla T_{k}\left(v_{j}\right)\right] d x \\
\quad+\int_{\left\{\left|u_{n}\right|>k\right\}} a\left(x, u_{n}, \nabla u_{n}\right) \nabla w_{n, j}^{h} d x .
\end{aligned}
$$

Set $m=4 k+h$. By using $\left(A_{3}\right)$ and the fact that $\nabla w_{n, j}^{h}=0$ if $\left|u_{n}(x)\right|>m$, we get

$$
\begin{aligned}
\int_{\left\{\left|u_{n}\right|>k\right\}} a\left(x, u_{n}, \nabla u_{n}\right) \nabla w_{n, j}^{h} d x & \\
& \geq-\int_{\left\{\left|u_{n}\right|>k\right\}}\left|a\left(x, T_{m}\left(u_{n}\right), \nabla T_{m}\left(u_{n}\right)\right)\right|\left|\nabla v_{j}\right| d x .
\end{aligned}
$$

Since $\left(\left|a\left(x, T_{m}\left(u_{n}\right), \nabla T_{m}\left(u_{n}\right)\right)\right|\right)_{n}$ is a bounded sequence in $L_{\bar{M}}(\Omega)$, for some subsequence still denoted $u_{n}$ and for some $l_{m} \in L_{\bar{M}}(\Omega)$ we have

$$
\left|a\left(x, T_{m}\left(u_{n}\right), \nabla T_{m}\left(u_{n}\right)\right)\right| \rightarrow l_{m} \quad \text { in } L_{\bar{M}}(\Omega) \text { for } \sigma\left(L_{\bar{M}}(\Omega), E_{M}(\Omega)\right)
$$

as $n \rightarrow \infty$, and since $\nabla v_{j} \chi_{\left\{\left|u_{n}\right|>k\right\}} \rightarrow \nabla v_{j} \chi_{\{|u|>k\}}$ strongly in $E_{M}(\Omega)$ as $n \rightarrow \infty$, we get

$$
-\int_{\left\{\left|u_{n}\right|>k\right\}}\left|a\left(x, T_{m}\left(u_{n}\right), \nabla T_{m}\left(u_{n}\right)\right)\right|\left|\nabla v_{j}\right| d x=-\int_{\{|u|>k\}} l_{m}\left|\nabla v_{j}\right| d x+\varepsilon(n) .
$$

We let $j \rightarrow \infty$ to obtain

$$
-\int_{\{|u|>k\}} l_{m}\left|\nabla v_{j}\right| d x=-\int_{\{|u|>k\}} l_{m}\left|\nabla T_{k}(u)\right| d x+\varepsilon(n, j)=\varepsilon(n, j) .
$$

Combining (4.27)-(4.29), we deduce

$$
\begin{aligned}
\int_{\Omega} a\left(x, u_{n}, \nabla u_{n}\right) \nabla w_{n, j}^{h} d x & \\
\geq & \int_{\Omega} a\left(x, T_{k}\left(u_{n}\right), \nabla T_{k}\left(u_{n}\right)\right)\left[\nabla T_{k}\left(u_{n}\right)-\nabla T_{k}\left(v_{j}\right)\right] d x \\
& +\varepsilon(n, h)+\varepsilon(n, j)+\varepsilon_{h}(n, j),
\end{aligned}
$$


which implies that

$$
\begin{aligned}
& \int_{\Omega} a\left(x, u_{n}, \nabla u_{n}\right) \nabla w_{n, j}^{h} d x \\
& \geq \int_{\Omega}\left[a\left(x, T_{k}\left(u_{n}\right), \nabla T_{k}\left(u_{n}\right)\right)-a\left(x, T_{k}\left(u_{n}\right), \nabla T_{k}\left(v_{j}\right) \chi_{s}^{j}\right)\right] \\
& \quad \times\left[\nabla T_{k}\left(u_{n}\right)-\nabla T_{k}\left(v_{j}\right) \chi_{s}^{j}\right] d x \\
& +\int_{\Omega} a\left(x, T_{k}\left(u_{n}\right), \nabla T_{k}\left(v_{j}\right) \chi_{s}^{j}\right)\left[\nabla T_{k}\left(u_{n}\right)-\nabla T_{k}\left(v_{j}\right) \chi_{s}^{j}\right] d x \\
& \quad-\int_{\Omega \backslash \Omega_{s}^{j}} a\left(x, T_{k}\left(u_{n}\right), \nabla T_{k}\left(u_{n}\right)\right) \nabla T_{k}\left(v_{j}\right) d x \\
& +\varepsilon(n, h)+\varepsilon(n, j)+\varepsilon_{h}(n, j)
\end{aligned}
$$

where $\chi_{s}^{j}$ is the characteristic function of $\Omega_{s}^{j}=\left\{x \in \Omega:\left|\nabla T_{k}\left(v_{j}\right)\right| \leq s\right\}$.

By (4.18) and the fact that $\nabla T_{k}\left(v_{j}\right) \chi_{\Omega \backslash \Omega_{s}^{j}} \in\left(E_{M}(\Omega)\right)^{N}$, the third term on the right hand side of (4.30) tends to $\int_{\Omega} \varrho_{k} \nabla T_{k}\left(v_{j}\right) \chi_{\Omega \backslash \Omega_{s}^{j}} d x$ as $n \rightarrow \infty$. Letting $j \rightarrow \infty$ we obtain, by (4.15),

$$
\int_{\Omega} \varrho_{k} \nabla T_{k}\left(v_{j}\right) \chi_{\Omega \backslash \Omega_{s}^{j}} d x=\int_{\Omega \backslash \Omega_{s}} \varrho_{k} \nabla T_{k}(u) d x+\varepsilon(n, j) .
$$

The second term on the right hand side of (4.30) goes to zero as first $n \rightarrow \infty$ and then $j \rightarrow \infty$. Indeed, since $a\left(x, T_{k}\left(u_{n}\right), \nabla T_{k}\left(v_{j}\right) \chi_{s}^{j}\right) \rightarrow$ $a\left(x, T_{k}(u), \nabla T_{k}\left(v_{j}\right) \chi_{s}^{j}\right)$ strongly in $\left(E_{\bar{M}}(\Omega)\right)^{N}$ by using $\left(A_{2}\right)$ and the Lebesgue theorem while $\nabla T_{k}\left(u_{n}\right) \rightarrow \nabla T_{k}(u)$ in $\left(L_{M}(\Omega)\right)^{N}$, we have

$$
\begin{aligned}
\int_{\Omega} a\left(x, T_{k}\left(u_{n}\right), \nabla T_{k}\left(v_{j}\right) \chi_{s}^{j}\right)\left[\nabla T_{k}\left(u_{n}\right)-\nabla T_{k}\left(v_{j}\right) \chi_{s}^{j}\right] d x \\
\quad=\int_{\Omega} a\left(x, T_{k}(u), \nabla T_{k}\left(v_{j}\right) \chi_{s}^{j}\right)\left[\nabla T_{k}(u)-\nabla T_{k}\left(v_{j}\right) \chi_{s}^{j}\right] d x+\varepsilon(n) .
\end{aligned}
$$

Letting $j \rightarrow \infty$, one has

$$
\begin{aligned}
\int_{\Omega} a\left(x, T_{k}(u), \nabla T_{k}\left(v_{j}\right) \chi_{s}^{j}\right)\left[\nabla T_{k}(u)-\nabla T_{k}\left(v_{j}\right) \chi_{s}^{j}\right] d x \\
=\int_{\Omega} a\left(x, T_{k}(u), \nabla T_{k}(u) \chi_{s}\right)\left[\nabla T_{k}(u)-\nabla T_{k}(u) \chi_{s}\right] d x+\varepsilon(j) .
\end{aligned}
$$

Finally,

$$
\int_{\Omega} a\left(x, T_{k}\left(u_{n}\right), \nabla T_{k}\left(v_{j}\right) \chi_{s}^{j}\right)\left[\nabla T_{k}\left(u_{n}\right)-\nabla T_{k}\left(v_{j}\right) \chi_{s}^{j}\right] d x=\varepsilon_{m}(n, j) .
$$


Combining (4.30)-(4.32), we deduce

$$
\begin{aligned}
& \int_{\Omega} a\left(x, u_{n}, \nabla u_{n}\right) \nabla w_{n, j}^{h} d x \\
& \geq \int_{\Omega}\left[a\left(x, T_{k}\left(u_{n}\right), \nabla T_{k}\left(u_{n}\right)\right)-a\left(x, T_{k}\left(u_{n}\right), \nabla T_{k}\left(v_{j}\right) \chi_{s}^{j}\right)\right] \\
& \quad \times\left[\nabla T_{k}\left(u_{n}\right)-\nabla T_{k}\left(v_{j}\right) \chi_{s}^{j}\right] d x-\int_{\Omega \backslash \Omega_{s}} \varrho_{k} \nabla T_{k}(u) d x+\varepsilon(n, j, h) .
\end{aligned}
$$

On the other hand, by using Proposition 4.2(ii) and (4.15) we can easily find that

$$
\int_{\Omega} \phi\left(u_{n}\right) \nabla w_{n, j}^{h} d x=\int_{\Omega} \phi(u) \nabla T_{2 k}\left(u-T_{h}(u)\right) d x+\varepsilon_{h}(n, j)=\varepsilon_{h}(n, j)
$$

and

$$
\int_{\Omega} F \nabla w_{n, j}^{h} d x=\int_{\Omega} F \nabla T_{2 k}\left(u-T_{h}(u)\right) d x+\varepsilon(n, j) .
$$

Combining (4.26) and (4.33)-(4.35), we get

$$
\begin{array}{r}
\int_{\Omega}\left[a\left(x, T_{k}\left(u_{n}\right), \nabla T_{k}\left(u_{n}\right)\right)-a\left(x, T_{k}\left(u_{n}\right), \nabla T_{k}\left(v_{j}\right) \chi_{s}^{j}\right)\right] \\
\times\left[\nabla T_{k}\left(u_{n}\right)-\nabla T_{k}\left(v_{j}\right) \chi_{s}^{j}\right] d x \\
\leq \int_{\Omega \backslash \Omega_{s}} \varrho_{k} \nabla T_{k}(u) d x+\int_{\Omega} F \nabla T_{2 k}\left(u-T_{h}(u)\right) d x+\varepsilon(n, j, h) .
\end{array}
$$

Now, we remark that

$$
\begin{aligned}
\int_{\Omega}\left[a\left(x, T_{k}\left(u_{n}\right), \nabla T_{k}\left(u_{n}\right)\right)-a\left(x, T_{k}\left(u_{n}\right), \nabla T_{k}(u) \chi_{s}\right)\right]\left[\nabla T_{k}\left(u_{n}\right)-\nabla T_{k}(u) \chi_{s}\right] d x \\
-\int_{\Omega}\left[a\left(x, T_{k}\left(u_{n}\right), \nabla T_{k}\left(u_{n}\right)\right)-a\left(x, T_{k}\left(u_{n}\right), \nabla T_{k}\left(v_{j}\right) \chi_{s}^{j}\right)\right] \\
\times\left[\nabla T_{k}\left(u_{n}\right)-\nabla T_{k}\left(v_{j}\right) \chi_{s}^{j}\right] d x \\
=\int_{\Omega} a\left(x, T_{k}\left(u_{n}\right), \nabla T_{k}\left(v_{j}\right) \chi_{s}^{j}\right)\left[\nabla T_{k}\left(u_{n}\right)-\nabla T_{k}\left(v_{j}\right) \chi_{s}^{j}\right] d x \\
-\int_{\Omega} a\left(x, T_{k}\left(u_{n}\right), \nabla T_{k}(u) \chi_{s}\right)\left[\nabla T_{k}\left(u_{n}\right)-\nabla T_{k}(u) \chi_{s}\right] d x \\
+\int_{\Omega} a\left(x, T_{k}\left(u_{n}\right), \nabla T_{k}\left(u_{n}\right)\right)\left[\nabla T_{k}\left(v_{j}\right) \chi_{s}^{j}-\nabla T_{k}(u) \chi_{s}\right] d x,
\end{aligned}
$$

and, as can be easily seen, each integral on the right hand side is of the form 
$\varepsilon(n, j)$, implying that

$$
\begin{aligned}
\int_{\Omega}\left[a \left(x, T_{k}\left(u_{n}\right),\right.\right. & \left.\left.\nabla T_{k}\left(u_{n}\right)\right)-a\left(x, T_{k}\left(u_{n}\right), \nabla T_{k}(u) \chi_{s}\right)\right] \\
& \times\left[\nabla T_{k}\left(u_{n}\right)-\nabla T_{k}(u) \chi_{s}\right] d x \\
= & \int_{\Omega}\left[a\left(x, T_{k}\left(u_{n}\right), \nabla T_{k}\left(u_{n}\right)\right)-a\left(x, T_{k}\left(u_{n}\right), \nabla T_{k}\left(v_{j}\right) \chi_{s}^{j}\right)\right] \\
& \times\left[\nabla T_{k}\left(u_{n}\right)-\nabla T_{k}\left(v_{j}\right) \chi_{s}^{j}\right] d x+\varepsilon(n, j),
\end{aligned}
$$

and thanks to (4.36) and (4.37), we deduce

$$
\begin{array}{r}
\int_{\Omega}\left[a\left(x, T_{k}\left(u_{n}\right), \nabla T_{k}\left(u_{n}\right)\right)-a\left(x, T_{k}\left(u_{n}\right), \nabla T_{k}(u) \chi_{s}\right)\right]\left[\nabla T_{k}\left(u_{n}\right)-\nabla T_{k}(u) \chi_{s}\right] d x \\
\leq \int_{\Omega \backslash \Omega_{s}} \varrho_{k} \nabla T_{k}(u) d x+\int_{\Omega} F \nabla T_{2 k}\left(u-T_{h}(u)\right) d x+\varepsilon(n, j, h) .
\end{array}
$$

Hence,

$$
\begin{aligned}
\limsup _{n \rightarrow \infty} \int_{\Omega}\left[a\left(x, T_{k}\left(u_{n}\right), \nabla T_{k}\left(u_{n}\right)\right)-a\left(x, T_{k}\left(u_{n}\right), \nabla T_{k}(u) \chi_{s}\right)\right] \\
\times\left[\nabla T_{k}\left(u_{n}\right)-\nabla T_{k}(u) \chi_{s}\right] d x \\
\leq \int_{\Omega \backslash \Omega_{s}} \varrho_{k} \nabla T_{k}(u) d x+\int_{\Omega} F \nabla T_{2 k}\left(u-T_{h}(u)\right) d x+\lim _{n \rightarrow \infty} \varepsilon(n, j, h) .
\end{aligned}
$$

We shall now prove that

$$
\int_{\Omega} F \nabla T_{2 k}\left(u-T_{h}(u)\right) d x \rightarrow 0 \quad \text { as } h \rightarrow \infty .
$$

If we take $u_{n}-T_{2 k}\left(u_{n}-T_{h}\left(u_{n}\right)\right)$ as a test function in (4.1) we obtain

$$
\begin{aligned}
\int_{\left\{h \leq\left|u_{n}\right| \leq 2 k+h\right\}} a\left(x, u_{n}, \nabla u_{n}\right) \nabla u_{n} d x+\int_{\Omega} \phi\left(u_{n}\right) \nabla T_{2 k}\left(u_{n}-T_{h}\left(u_{n}\right)\right) d x \\
\leq \int_{\left\{h \leq\left|u_{n}\right| \leq 2 k+h\right\}} F \nabla u_{n} d x+\int_{\Omega} f_{n} T_{2 k}\left(u_{n}-T_{h}\left(u_{n}\right)\right) d x .
\end{aligned}
$$

Since $\int_{\Omega} \phi\left(u_{n}\right) \nabla T_{2 k}\left(u_{n}-T_{h}\left(u_{n}\right)\right) d x=0$, we get

$$
\begin{aligned}
\int_{\left\{h \leq\left|u_{n}\right| \leq 2 k+h\right\}} a\left(x, u_{n}, \nabla u_{n}\right) \nabla u_{n} d x \\
\quad \leq \int_{\left\{h \leq\left|u_{n}\right| \leq 2 k+h\right\}} F \nabla u_{n} d x+\int_{\Omega} f_{n} T_{2 k}\left(u_{n}-T_{h}\left(u_{n}\right)\right) d x,
\end{aligned}
$$

which yields, thanks to $\left(A_{4}\right)$ and Young's inequality, $\frac{\alpha}{2} \int_{\left\{h \leq\left|u_{n}\right| \leq 2 k+h\right\}} M\left(\left|\nabla u_{n}\right|\right) d x \leq C_{1} \int_{\left\{\left|u_{n}\right|>h\right\}} \bar{M}(|F|) d x+\int_{\Omega} f_{n} T_{2 k}\left(u_{n}-T_{h}\left(u_{n}\right)\right) d x$. 
Letting $n \rightarrow \infty$, by using the Fatou lemma, we get

$$
\frac{\alpha}{2} \int_{\{h \leq|u| \leq 2 k+h\}} M(|\nabla u|) d x \leq C_{1}(k) \int_{\{|u|>h\}} \bar{M}(|F|) d x+\int_{\{|u|>h\}}|f| d x .
$$

Consequently,

$$
\limsup _{h \rightarrow \infty} \int_{\{h \leq|u| \leq 2 k+h\}} M(|\nabla u|) d x=0
$$

so that

$$
\lim _{h \rightarrow \infty} \int_{\Omega} F \nabla T_{2 k}\left(u-T_{h}(u)\right) d x=0,
$$

which implies (4.39).

Thanks to (4.38) and (4.39), we can write

$$
\begin{aligned}
& \limsup _{n \rightarrow \infty} \int_{\Omega}\left[a\left(x, T_{k}\left(u_{n}\right), \nabla T_{k}\left(u_{n}\right)\right)-\right.\left.a\left(x, T_{k}\left(u_{n}\right), \nabla T_{k}(u) \chi_{s}\right)\right] \\
& \times\left[\nabla T_{k}\left(u_{n}\right)-\nabla T_{k}(u) \chi_{s}\right] d x \\
& \leq \int_{\Omega \backslash \Omega_{s}} \varrho_{k} \nabla T_{k}(u) d x+\lim _{n \rightarrow \infty} \varepsilon(n, j, h),
\end{aligned}
$$

in which we can pass to the limit as $j, h, s \rightarrow \infty$ to obtain

$$
\begin{aligned}
& \limsup _{s \rightarrow \infty} \limsup _{n \rightarrow \infty} \int_{\Omega}\left[a\left(x, T_{k}\left(u_{n}\right), \nabla T_{k}\left(u_{n}\right)\right)-a\left(x, T_{k}\left(u_{n}\right), \nabla T_{k}(u) \chi_{s}\right)\right] \\
& \times\left[\nabla T_{k}\left(u_{n}\right)-\nabla T_{k}(u) \chi_{s}\right] d x=0 .
\end{aligned}
$$

Finally, Lemma 4.1 yields the conclusion of Proposition 4.4.

4.3. Proof of Theorem 3.1. Let $v \in K_{\psi} \cap W_{0}^{1} E_{M}(\Omega) \cap L^{\infty}(\Omega)$. Taking $u_{n}-T_{k}\left(u_{n}-v\right)$ as a test function in (4.1), we can write

$$
\begin{aligned}
\int_{\Omega} a\left(x, u_{n}, \nabla u_{n}\right) \nabla T_{k}\left(u_{n}-v\right) d x+\int_{\Omega} \phi\left(T_{n}\left(u_{n}\right)\right) \nabla T_{k}\left(u_{n}-v\right) d x \\
\leq \int_{\Omega} f_{n} T_{k}\left(u_{n}-v\right) d x+\int_{\Omega} F_{n} \nabla T_{k}\left(u_{n}-v\right) d x,
\end{aligned}
$$

which implies that

$$
\begin{aligned}
& \int_{\left\{\left|u_{n}-v\right| \leq k\right\}} a\left(x, u_{n}, \nabla u_{n}\right) \nabla u_{n} d x \\
& \quad-\int_{\left\{\left|u_{n}-v\right| \leq k\right\}} a\left(x, T_{k+\|v\|_{\infty}}\left(u_{n}\right), \nabla T_{k+\|v\|_{\infty}}\left(u_{n}\right)\right) \nabla v d x \\
& \quad+\int_{\Omega} \phi\left(T_{k+\|v\|_{\infty}}\left(u_{n}\right)\right) \nabla T_{k}\left(u_{n}-v\right) d x \\
& \leq \int_{\Omega} f_{n} T_{k}\left(u_{n}-v\right) d x+\int_{\Omega} F_{n} \nabla T_{k}\left(u_{n}-v\right) d x .
\end{aligned}
$$


By Fatou's lemma and the fact that

$$
a\left(x, T_{k+\|v\|_{\infty}}\left(u_{n}\right), \nabla T_{k+\|v\|_{\infty}}\left(u_{n}\right)\right) \rightarrow a\left(x, T_{k+\|v\|_{\infty}}(u), \nabla T_{k+\|v\|_{\infty}}(u)\right)
$$

weakly in $\left(L_{\bar{M}}(\Omega)\right)^{N}$ for $\sigma\left(\prod L_{\bar{M}}, \prod E_{M}\right)$ one easily sees that

$$
\begin{aligned}
\lim _{n \rightarrow \infty}\{ & \int_{\left\{\left|u_{n}-v\right| \leq k\right\}} a\left(x, u_{n}, \nabla u_{n}\right) \nabla u_{n} d x \\
& \left.-\int_{\left\{\left|u_{n}-v\right| \leq k\right\}} a\left(x, T_{k+\|v\|_{\infty}}\left(u_{n}\right), \nabla T_{k+\|v\|_{\infty}}\left(u_{n}\right)\right) \nabla v d x\right\} \\
\geq & \int_{\{|u-v| \leq k\}} a(x, u, \nabla u) \nabla u d x \\
& -\int_{\{|u-v| \leq k\}} a\left(x, T_{k+\|v\|_{\infty}}(u), \nabla T_{k+\|v\|_{\infty}}(u)\right) \nabla v d x .
\end{aligned}
$$

On the other hand, by using Proposition 4.2, we can easily see that

$$
\begin{aligned}
\int_{\Omega} \phi\left(T_{n}\left(u_{n}\right)\right) \nabla T_{k}\left(u_{n}-v\right) d x & \rightarrow \int_{\Omega} \phi(u) \nabla T_{k}(u-v) d x, \\
\int_{\Omega} F \nabla T_{k}\left(u_{n}-v\right) d x & \rightarrow \int_{\Omega} F \nabla T_{k}(u-v) d x, \\
\int_{\Omega} f_{n} T_{k}\left(u_{n}-v\right) d x & \rightarrow \int_{\Omega} f T_{k}(u-v) d x
\end{aligned}
$$

as $n \rightarrow \infty$.

Combining (4.40)-(4.44), we have

$$
\begin{array}{r}
\int_{\Omega} a(x, u, \nabla u) \nabla T_{k}(u-v) d x+\int_{\Omega} \phi(u) \nabla T_{k}(u-v) d x \\
\leq \int_{\Omega} f T_{k}(u-v) d x+\int_{\Omega} F \nabla T_{k}(u-v) d x \\
\quad \forall v \in K_{\psi} \cap W_{0}^{1} E_{M}(\Omega) \cap L^{\infty}(\Omega) .
\end{array}
$$

Now, let $v \in K_{\psi} \cap L^{\infty}(\Omega)$. By $\left(A_{5}\right)$ there exists $v_{j} \in K_{\psi} \cap W_{0}^{1} E_{M}(\Omega) \cap L^{\infty}(\Omega)$ such that $v_{j}$ converges to $v$ in the modular sense. Let $h>\max \left(\left\|v_{0}\right\|_{\infty},\|v\|_{\infty}\right)$. Taking $v=T_{h}\left(v_{j}\right)$ in (4.45), we have

$$
\begin{aligned}
\int_{\Omega} a(x, u, \nabla u) \nabla T_{k}(u- & \left.T_{h}\left(v_{j}\right)\right) d x+\int_{\Omega} \phi(u) \nabla T_{k}\left(u-T_{h}\left(v_{j}\right)\right) d x \\
& \leq \int_{\Omega} f T_{k}\left(u-T_{h}\left(v_{j}\right)\right) d x+\int_{\Omega} F \nabla T_{k}\left(u-T_{h}\left(v_{j}\right)\right) d x .
\end{aligned}
$$


We can easily pass to the limit as $j \rightarrow \infty$ to get

$$
\begin{aligned}
\int_{\Omega} a(x, & u, \nabla u) \nabla T_{k}\left(u-T_{h}(v)\right) d x+\int_{\Omega} \phi(u) \nabla T_{k}\left(u-T_{h}(v)\right) d x \\
& \leq \int_{\Omega} f T_{k}\left(u-T_{h}(v)\right) d x+\int_{\Omega} F \nabla T_{k}\left(u-T_{h}(v)\right) d x \quad \forall v \in K_{\psi} \cap L^{\infty}(\Omega) .
\end{aligned}
$$

Finally, since $h \geq \max \left(\left\|v_{0}\right\|_{\infty},\|v\|_{\infty}\right)$, we deduce

$$
\begin{aligned}
& \int_{\Omega} a(x, u, \nabla u) \nabla T_{k}(u-v) d x+\int_{\Omega} \phi(u) \nabla T_{k}(u-v) d x \\
& \quad \leq \int_{\Omega} f T_{k}(u-v) d x+\int_{\Omega} F \nabla T_{k}\left(u-T_{h}(v)\right) d x \quad \forall v \in K_{\psi} \cap L^{\infty}(\Omega), \forall k>0 .
\end{aligned}
$$

Thus, the proof of Theorem 3.1 is complete.

\section{References}

[1] R. Adams, Sobolev Spaces, Academic Press, New York, 1975.

[2] L. Aharouch, A. Benkirane and M. Rhoudaf, Strongly nonlinear elliptic variational unilateral problems in Orlicz space, Abstr. Appl. Anal. 2006, 46867, 20 pp.

[3] L. Aharouch and M. Rhoudaf, Strongly nonlinear ellitptic unilateral problems in Orlicz space and $L^{1}$ data, J. Inequal. Pure Appl. Math. 6 (2005), no. 2, art. 54, 20 pp.

[4] P. Bénilan, L. Boccardo, T. Gallouët, R. Gariepy, M. Pierre and J. L. Vázquez, An $L^{1}$-theory of existence and uniqueness of nonlinear elliptic equations, Ann. Scuola Norm. Sup. Pisa 22 (1995), 240-273.

[5] A. Benkirane and J. Bennouna, Existence of entropy solutions for nonlinear problems in Orlicz spaces, Abstr. Appl. Anal. 7 (2002), no. 2, 85-102.

[6] - - - Existence and uniqueness of solution of unilateral problems with $L^{1}$-data in Orlicz spaces, Ital. J. Pure Appl. Math. 16 (2004), 87-102.

[7] A. Benkirane and A. Elmahi, A strongly nonlinear elliptic equation having natural growth terms and $L^{1}$ data, Nonlinear Anal. 39 (2000), 403-411.

[8] L. Boccardo, Some nonlinear Dirichlet problem in $L^{1}$ involving lower order terms in divergence form, in: Progress in Elliptic and Parabolic Partial Differential Equations (Capri, 1994), Pitman Res. Notes Math. Ser. 350, Longman, Harlow, 1996, 43-57.

[9] L. Boccardo and G. R. Cirmi, Existence and uniqueness of solution of unilateral problems with $L^{1}$ data, J. Convex Anal. 6 (1999), 195-206.

[10] G. Dal Maso, F. Murat, L. Orsina and A. Prignet, Renormalized solutions of elliptic equations with general measure data, Ann. Scuola Norm. Sup. Pisa Cl. Sci. 28 (1999), 741-808.

[11] A. Elmahi and D. Meskine, Existence of solutions for elliptic problems having natural growth terms in Orlicz spaces, Abstr. Appl. Anal. 12 (2004), 1031-1045.

[12] - - - Nonlinear elliptic problems having natural growth and $L^{1}$ data in Orlicz spaces, Ann. Mat. Pura Appl. 184 (2005), 161-184.

[13] J. P. Gossez, Nonlinear elliptic boundary value problems for equations with rapidly (or slowly) increasing coefficients, Trans. Amer. Math. Soc. 190 (1974), 163-205. 
[14] J. P. Gossez and V. Mustonen, Variational inequalities in Orlicz-Sobolev spaces, Nonlinear Anal. 11 (1987), 379-492.

[15] M. A. Krasnosel'skiı̌ and Ya. B. Rutickiı̌, Convex Functions and Orlicz Spaces, Noordhoff, Groningen, 1969.

Département de Mathématiques et Informatique

Faculté des Sciences Dhar-Mahraz

B.P. 1796 Atlas, Fès, Maroc

E-mail: 1_aharouch@yahoo.fr

azroul_elhoussine@yahoo.fr rhoudaf_mohamed@yahoo.fr

Received on 14.11.2005;

revised version on 27.6.2006 
